\title{
SERVICIOS SOCIALES DE LA SEGURIDAD SOCIAL. ASPECTOS INSTITUCIONALES
}

\section{DEMETRIO CASADO}

Técnico Superior de la Administración de la Seguridad Social.

\section{INTRODUCCIÓN}

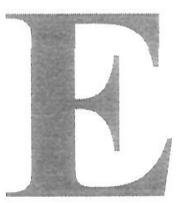

n el año que la Unión Europea dedica a las personas con discapacidad, parece oportuno reseñar y analizar los Servicios Sociales de la Seguridad Social destinados a esa faceta de la condición humana. Voy a enfocarlos desde el punto de vista institucional, por parecerme que no es muy conocido. Este enfoque me lleva a extender mi examen a otras instituciones del gran sistema de protección social, que se entrelazan con dichos Servicios Sociales del área de la discapacidad.

El aislamiento político y cultural de la primera parte del franquismo no fue óbice para que los rectores de la que se llamaba Previsión Social española conocieran y asumieran los avances llevados a cabo en los países industrializados. Los Seguros Sociales obligatorios y garantizados eran, sin duda, la pieza clave del Estado de bienestar que se estaba construyendo a gran ritmo en la Europa occidental tras la segunda guerra mundial. Por lo demás, a finales del decenio de los 50 se inicia en España un despegue económico que se consolidaría en los años 60: el "milagro económico español". Este proceso era gestionado por la "sección" económica del Gobierno, de orientación capitalista y tecnocrática, en tanto que la política laboral y de previsión social continuaba, a la sazón, en manos de ministros falangistas, sucesores de Girón.

\section{LA LEY DE BASES DE 1963: PRESTACIONES Y COMPLE- MENTOS}

El contexto desarrollista de los años 60 debió de ser circunstancia propicia para la ampliación de la previsión social impulsada por la línea nacionalsindicalista del régimen franquista. Sirvan como referencia de la tendencia expansiva de la cobertura personal de la Seguridad Social en aquellos años estas dos cifras (en miles) de acogidos al Seguro de 
Enfermedad: 3.784 , en 1955 , y 6.224 , en $1965 .^{\prime}$

El salto hacia adelante de la previsión social se inicia mediante la Ley 193/1963, de 28 de diciembre, de Bases de la Seguridad Social. Esta nueva denominación venía justificada por cambios importantes. ${ }^{2}$ Por de pronto, se amplía el campo personal de aplicación de la acción protectora (base segunda) hasta desbordar su inicial perímetro selectivo de signo redistribuidor. Y el contenido de la acción protectora se establece en estos términos (base quinta, 8):

"a) La asistencia sanitaria en los casos de maternidad, de enfermedad, común o profesional, y de accidentes, sean o no de trabajo.

b) Las prestaciones económicas en los supuestos de incapacidad laboral transitoria, invalidez, vejez, desempleo, muerte y supervivencia, así como las que se otorguen en contingencias y situaciones especiales que se regulen.

c) El régimen de protección a la familia.

d) Los Servicios Sociales que dentro de los límites de los recursos financieros le corresponda asumir o le fueran asignados en materia de asistencia, medicina preventiva, higiene y seguridad del trabajo, reeducación y rehabilitación de inválidos, empleo o colocación, promoción social y en aquellas otras en que el establecimiento de tales Servicios se considere conveniente o resulte necesario por exigencias de una más adecuada coordinación administrativa..."

Las acciones a), b) y c) son el núcleo de la Seguridad Social, que sería regulado mediante el Texto Articulado de la Ley de Bases, aprobado por el Decreto 907/1966, de 21 de abril. Posteriormente, les afectaría la Ley 24/1972, de 21 de junio, de financiación y perfeccionamiento de la acción protectora. Ambas leyes serían objeto del Texto Refundido de la Ley General de la Seguridad Social, aprobado por el Decreto 2.064/1974, de 30 de mayo. Dejando aparte problemas de implementación -especialmente en lo que se refiere a la protección familiar-, este bloque vino a ser una forma de avance e integración de los Seguros Sociales, de modo que se presta a ser caracterizado en su conjunto. El ámbito subjetivo, como quedó dicho, pasa a ser masivo. Su objeto y sus medios son mixtos: la necesidad sanitaria, que viene a ser correspondida por la asistencia médico-farmacéutica; la interrupción o el cese del ingreso por el trabajo resultantes de una amplia gama de contingencias, que son afrontadas con prestaciones económicas; las

Instituto Nacional de Estadistica, España, panorámica social, 1974, Madrid, 1975, p. 135.

2 Puede verse un propuesta de los rasgos del cambio de la previsión a la seguridad social en Manuel Alonso Olea, "La Seguridad Social: pasado, presente y futuro", en Bernardo Gonzalo González y Magdalena Nogueira Gustavino, Cien años de Seguridad Social, Fraternidad-Muprespa y UNED, Madrid, 2000, p. 168. 
llamadas cargas familiares, que dan lugar a ciertas ayudas regladas. Sin perjuicio de algunas aportaciones del Estado, la financiación es contributiva, de modo que el régimen de provisión de este núcleo prestacional es de carácter previsor y adquisitivo. La técnica protectora que se aplica consiste en la creación de derechos subjetivos. Su gestión y exigibilidad conlleva algún problema en las prestaciones mediadas por juicios facultativos (prescripción médica, valoración de incapacidad), pero ello no afecta a la identidad de la técnica protectora.

Los complementos del inciso d) son ampliaciones novedosas y atípicas, pero no dejan de contar con ciertos antecedentes. El Seguro de Maternidad instituido en 1929 vino a prever ingresos de sustitución en el periodo de descanso maternal; esta es una prestación típica de un seguro. Pero el Real Decreto-ley por el que se instituye abre paso a otras acciones y servicios sanitarios y sociales materno-infantiles complementarios. ${ }^{3}$ En otro orden de cosas, la sección nacionalsindicalista del régimen adoptó el concepto de previsión social ofensiva, de la que pueden servir como muestra las Universidades Laborales. Su labor técnica vino a consistir en producir enseñanzas regladas -con una estimable guarnición educativa- a los hijos becarios de los mutualistas, pero su creador, el ministro Girón, las concibió inicialmente para servir al ascenso de la clase trabajadora al liderazgo social y político. Esta era su idea: "Para salvar a la clase trabajadora de la ignorancia y para darle la oportunidad de entrar ella misma en el Estado y dirigirle con el mismo derecho que cualquier otra clase social y para garantizarle que jamás la clase dirigente iba a abusar de su debilidad económica y de su indigencia cultural, no había más remedio que el remedio heroico: hacer de la clase trabajadora también clase dirigente y arrebatar no por la fuerza ni por la subversión, no por el disturbio y el desorden, no por la tragedia y la guerra sino por la fuerza de la razón, por la luz de la inteligencia y por la paz el cetro del mando de la sociedad a una sola mano, a una sola clase y ofrecérselo a la sociedad entera sin distinción ni privilegio. Para esto Franco sólo vio el camino de la Cultura entendida como patrimonio universal igual que el aire que el hombre respira." ${ }^{4}$

En el inciso d) se menciona la materia "asistencia" como una de las

Ver Manuel Aznar López, "Una institución centenaria: La Seguridad Social Española", en Revista del Ministerio de Trabajo y Asuntos Sociales. Seguridad Social, $\mathrm{n}^{\circ} 24,2000$, p. 24 .

Mensaje del ministro José Antonio Girón de Velasco a las Universidades de Sevilla, Códoba y Tarragona al iniciar las mismas sus actividades académicas en noviembre de 1956. Citado por Francisco Aguilar y Paz, "Universidades Laborales": creación y propósitos fundacionales", en Las Universidades Laborales: evolución, situación y perspectivas, Instituto de Técnicas Educativas de Universidades Laborales, Alcalá de Henares, 1977, multicopiado, pp. 10 y 11. 
posibles de los Servicios Sociales, pero la propia Ley previene más adelante (base decimosexta, 67) un "régimen de asistencia", en estos términos: "La Seguridad Social, con cargo a fondos que a tal efecto se determinen, podrá dispensar a las personas incluidas en su campo de aplicación y a los familiares o asimilados que de ellas dependan, los servicios y auxilios económicos que en atención a estados y situaciones de necesidad, se consideren precisos previa demostración, salvo en casos de urgencia, de que el interesado carece de los recursos indispensables para hacer frente a tales estados o situaciones." El Texto Articulado se ciñe a esta concepción, que no sería afectada por la Ley de 1972, de modo que cabe abordar su caracterización institucional. Ya se ve que el ámbito personal es selectivo por criterio de necesidad; la legislación no limita expresamente la misma al factor económico. En virtud de esto, hemos de asumir que los objetos y medios pueden ser varios. La provisión de los recursos es adquisitiva, por lo que atañe al sistema de la Seguridad Social, y adscriptiva en el trámite de concesión. Así, la técnica de protección, al contrario de la relativa a las prestaciones nucleares, no pasa de ser una habilitación administrativa a los gestores de la Seguridad Social para ciertas acciones como mucho discrecionales.

Paso a la otra figura complementaria, los Servicios Sociales. "Como complemento de las prestaciones otorgadas ante las diversas situaciones y contingencias" y buscando "la apertura hacia campos nuevos y fecundos de promoción social y comunitaria", según se dice en el preámbulo de la Ley (III.1), la misma franquea el paso a la creación de los Servicios Sociales. Según el texto trascrito antes (base quinta, 18,b), los campos objeto de acción no están limitados, si bien la propia Ley habilita estos cuatro Servicios Sociales (base decimoquinta): Higiene y Seguridad del Trabajo, Medicina Preventiva, Reeducación y Rehabilitación de Inválidos y Acción Formativa. El Texto refundido mantiene esta previsión, salvo que para el tercero adopta la denominación "Recuperación de inválidos".

\section{LOS SERVICIOS SOCIALES SUPERVIVIENTES}

Voy a examinar el proceso de construcción institucional de los Servicios Sociales que sobrevivieron hasta el presente. Comenzaré por el relativo a los "inválidos", del que acaso sea oportuno recordar que tuvo algún antecedente en la etapa de los Seguros Sociales. Al margen de los mismos, la Sanidad Nacional había tomado cartas en el problema de la invalidez mediante la creación, en 1949, del Patronato Nacional de Lucha contra la Invalidez, que sería sustituido por el de Rehabilita- 
ción y Recuperación de Inválidos, en 1949.5

La Ley de Bases de 1963 tomó un compromiso importante con las situaciones de enfermedad y derivadas de accidentes. Según vimos en el texto transcrito, se previó asistencia sanitaria tanto para la enfermedad profesional como para la común, y también para los accidentes de trabajo y los ajenos al mismo. Por otra parte, entre las prestaciones por invalidez, se incluyó el "derecho a tratamientos especializados de rehabilitación y de readaptación" (base octava) y, además, se previó el ya citado Servicio Social de Reeducación y Rehabilitación de Inválidos, con este contenido: "El régimen de la Seguridad Social organizará, con la amplitud necesaria, los Centros y Servicios de recuperación fisiológica, reeducación, readaptación y rehabilitación profesional de los trabajadores inválidos" (base decimoquinta). Aparentemente, esos Centros y Servicios habrían de proveer aquellos tratamientos especializados, en cuyo caso serían el instrumento de aquellas, y no un complemento de una de las prestaciones por invalidez ni, por lo mismo, un Servicio Social.

El Texto Articulado Primero de la Ley de Bases, aprobado por Decreto de 907/1966, de 21 de abril, trata las prestaciones recuperadoras como si tuvieran carácter indemnizatorio y no rehabilitador, pues las postpone a la declaración de invalidez (art. 139). En cuanto al Servicio Social de reeducación y rehabilitación de inválidos, la norma articulada lo trasforma en una institución doblemente selectiva, en cuanto que le encomienda la misión de cubrir, con carácter de asistencia social, casos excluidos del régimen ordinario de las prestaciones de reeducación y rehabilitación (art. 30). He aquí su regulación: "El Ministerio de Trabajo, coordinadamente con la Seguridad Social y en régimen de colaboración en su caso con la organización sindical o con otras Instituciones públicas, de la Iglesia o privadas, organizará los Servicios Sociales para extender la acción protectora, en línea de asistencia social, con el ritmo y alcance que permitan las disponibilidades financieras a las personas que carezcan del derecho previsto en el artículo anterior -a la reeducación y la rehabilitación-; señaladamente y entre otras a los inválidos permanentes que sean pensionistas de la Seguridad Social con anterioridad a la vigencia de la presente Ley" (art. 31). La opción institucional sustantiva -"extender la acción protectora"- de esta regulación se aparta de la concepción organizatoria o instrumental del Servicio Social de reeducación y rehabilitación de inválidos adoptada por la Ley de Bases, ${ }^{6}$ cabe pensar que con el buen propósito de salvar la señalada incoherencia en que la misma incurriera.

\footnotetext{
Ver Guia de actividades públicas asistenciales, Ministerio de Gobernación, Madrid,
} 1967, p. 178 y ss. 
Siguiendo en el nivel legal, el Texto Refundido de la Ley General de Seguridad Social, aprobado por Decreto 2065/1974, de 30 de mayo, en lo sustancial, deja las cosas como estaban por lo que atañe al Servicio Social de Reeducación y Rehabilitación de Inválidos, salvo que lo denomina de Recuperación de Inválidos (art. 25). Así había de ser, supuesto que no se había producido ninguna variación legislativa sobre el mismo que hubiera de ser refundida. Por cierto, tampoco se había producido ninguna novedad reglamentaria concerniente al Servicio Social. En el citado periodo se adoptaron medidas reglamentarias relativas a la invalidez - como la Orden de 15 de abril de 1969-, pero no incidieron en el Servicio Social de marras. De este modo, esa institución selectiva engendrada por la legislación de la Seguridad Social no había sido aún alumbrada a la fecha del citado Texto Refundido.

En el curso de esa demora, se crearía un Servicio Social no previsto expresamente en la normativa legal citada. La Ley de Bases comprometió, dentro de la protección a la familia, un tratamiento especial para las que hubiera hijos subnormales -terminología de la época- (base undécima). Esta previsión fue recogida por el Texto Articulado Primero (disposición final $4^{\mathrm{a}}$ ), y en él se fundó la creación, mediante Decreto de 20 de septiembre de 1968, del Servicio Social de Asistencia a los Menores Subnormales. Dicha norma fue modificada por otra de igual rango y fecha de 9 de abril de 1970 en el sentido de suprimir el límite de edad de los beneficiarios finales. Por Orden de 8 de mayo de ese año se aprueba el texto refundido de las normas reguladoras de asistencia de la Seguridad Social a los subnormales. Como se ve, se suprime la palabra "menores" en la denominación del Servicio Social. A la vista de su base jurídica, cabe preguntarse si a la nueva criatura se le asigna la denominación "Servicio Social" con propiedad, dentro del marco terminológico de la Seguridad Social. Otro asunto taxonómico que conviene señalar es que el término "subnormal" se emplea en la citada norma en sentido muy amplio.

En cuanto a la estructura institucional de este ente, es obvio su carácter selectivo; pero el criterio de protección no es la pobreza material u otra forma de precariedad, sino unas necesidades especiales que se le plantean a las familias con hijos afectos de la llamada subnormalidad. El objeto y los recursos previstos para la acción del Servicio fueron: 1) la concesión de "una aportación económica de mil quinientas pesetas mensuales, para contribuir al sostenimiento de los gastos que la educación, instrucción y recuperación de los menores

\footnotetext{
Ver Manuel Aznar López y Carlos Osuna Novel, “Antecedentes", en 10 Años del Servicio Social de Minusválidos, Instituto Nacional de Servicios Sociales, Madrid, 1983, pp. 20 y 21.
} 
subnormales origine a los familiares que los tengan a su cargo"; 2) la creación de centros para desarrollar esas funciones. De estos dos medios, la práctica de gestión consolidó el primero y soslayó el segundo; como, por lo demás, no se exigió la aplicación de la aportación económica a su fin, el Servicio Social se desnaturalizó. La provisión de los medios es adquisitiva, por el carácter contributivo de la Seguridad Social. En cuanto a la técnica de protección, el Decreto instituyente parece entrañar un compromiso de derechos subjetivos, el cual puede verse confirmado en la Orden de 22 de febrero de 1969 por la que se dictan normas de aplicación del Decreto.

El segundo Servicio Social de la Seguridad Social al que se dio existencia operativa fue el de Asistencia a Ancianos, creado por Orden de 19 de marzo de 1970. En este caso se tomó base jurídica en el carácter abierto de la enumeración de Servicios Sociales del Texto Articulado Primero de 1966 (art. 20.1d). La citada Orden define el ámbito subjetivo del Servicio Social de acuerdo, en términos generales, con su denominación (art. 3), de modo que tiene carácter selectivo. El criterio de selección tampoco es en este caso la pobreza u otra circunstancia de menesterosidad efectiva, sino las presuntas necesidades especiales relacionadas con la edad y/o con la condición de pensionista. La Orden prevé una gama o cartera de medios gerontológicos para el Servicio Social (ayuda a domicilio, centros de día, residencias y otros indeterminados). El régimen de provisión es, por su entronque financiero, adquisitivo. En cuanto a la técnica de protección, la clave viene dada por esta formulación de la norma citada: "El Servicio Social de Asistencia a los Ancianos podrá ejercer su acción mediante: (sigue la enumeración de medios)" (art. 2). No establece, pues, la norma derechos subjetivos de acceso a tales medios; no obliga a que la Seguridad Social los provea en cantidad y régimen de oferta determinados; así pues, como mucho, se trata de un mandato prestacional no determinado; como poco, estamos ante una mera habilitación administrativa para el servicio común -de los distintos regímenes de la Seguridad Social-que crea la propia Orden (art. 1.2).

El Servicio Social de Asistencia a los Ancianos fue rebautizado como de Asistencia a Pensionistas de la Seguridad Social con ocasión de la Orden de 5 de abril de 1974 (art. 1), cuyo objeto propio fue la aprobación del Estatuto de Personal del mismo. Se aprovechó también esa norma para explicitar las dimensiones orgánica y prestacional del Servicio: "con el carácter de un Servicio Común gestor de un Servicio Social de los previstos en el ap. d) del num. 1 del art. 20 de la Ley de Seguridad Social" (art.1). Se produciría un nuevo cambio de la denominación del Servicio, según se verá después. 
El Decreto 2531/1970, de 22 de mayo, sobre empleo de trabajadores minusválidos trajo una primera novedad consistente en la demarcación de un ámbito subjetivo más amplio que el de los trabajadores inválidos por el trabajo. En efecto, la norma toma como objeto de sus medidas a las "personas en edad laboral que estén afectadas en su capacidad física o psíquica en el grado que reglamentariamente se determine, sin que en ningún caso pueda ser inferior al treinta y tres por ciento, que les impida obtener o conservar empleo adecuado" (art. 1). Muy razonablemente, prevé que el grado de capacidad se determinará después de recibir los tratamientos recuperación (art. 2). El contenido sustantivo del Decreto consiste en "Medidas de recuperación, formación y empleo", que hacen parte de las que suelen denominarse políticas activas. En el orden orgánico, el Decreto crea un servicio común, que vincula a la Dirección General de Seguridad Social, y que denomina Servicio Social de Recuperación y de Rehabilitación de Minusválidos (art. 22.1). El cometido material que se prevé para el mismo, además de las acciones que se le asignen reglamentariamente, consiste en "principalmente las encaminadas a la rehabilitación médica y laboral".

El Servicio Social de Recuperación y Rehabilitación de Minusválidos (SEREM, según el acrónimo que se le adjudicara), tiene un nombre distinto, bien que parecido, al de Reeducación y Rehabilitación de Inválidos. Su ámbito subjetivo, como ya quedó indicado, es menos selectivo que el de éste. El contenido material es aparentemente coincidente. Su técnica de protección es muy diferente: aquel entrañaba un compromiso de derecho subjetivo, según la formulación del Texto Articulado Primero de 1966, en tanto que la norma que crea el SEREM no va más allá de habilitar a la Administración central para dotarse de un órgano de gestión. Esto último se verá confirmado por la Orden de 24 de noviembre de 1971, por la que se regula el SEREM, en la que se le asignan funciones de intervención indirecta y/o indeterminadas en relación a su exigibilidad y acaso no todas propias de la Seguridad Social (art. 2). Sí coinciden los dos entes comparados en lo que se refiere a su fuente financiera. Resumiendo: las diferencias relativas al ámbito subjetivo y a la naturaleza institucional parecen indicar que el SEREM no fue la encarnación del Servicio Social de Reeducación y Rehabilitación de Inválidos. Además, aún cabe plantearse si, a la vista de su fundamento jurídico y de sus funciones, era un Servicio Social de la Seguridad Social en sentido estricto.

Por medio del Decreto $7317 / 1974$, de 21 de febrero, se produce la fusión de los servicios comunes concernientes a subnormales y a minusválidos; prevaleció este último con la denominación Servicio Social de Recuperación y Rehabilitación de Minusválidos Físicos y Psíquicos, al que se le mantuvo el acrónimo SEREM. Por lo dicho ante- 
riormente, resulta obvio que se integraron dos servicios comunes responsables de sendos Servicios Sociales, estos de muy distinta naturaleza institucional. Cabe entender que esta circunstancia fue positiva en el orden práctico, en cuanto que las diferencias eran complementarias y su anexión vino a propiciar la construcción fáctica de una agencia única y referencial para los asuntos de la discapacidad. En un orden casuístico, cabe entender que el Servicio Social de Asistencia a Subnormales, mediante la capacidad del servicio común de su socio, vino a tener al fin posibilidad de construir aquellos centros previstos como segunda vía de su acción en la norma que lo instituyera.

Este epígrafe debe concluir reseñando el Real Decreto-Ley 36/ 1978, de 16 de noviembre, sobre gestión institucional de la Seguridad Social, la salud y el empleo. Para esa fecha ya había sido aprobada la Constitución en el Parlamento y estaba próxima a ser sometida a referendum. En el orden económico y social, las circunstancias eran muy distintas de las que propiciaron la creación de los Servicios Sociales de la Seguridad Social a partir de la Ley de Bases. La crisis económica mundial, coincidente con las fuertes subidas del precio del petróleo en 1971 y 73, se había instalado plenamente en España; su efecto deletéreo sobre el empleo pudo verse reforzado por unas reivindicaciones laborales que encontraron la mejor circunstancia política para manifestarse (el cambio de régimen) y la peor coyuntura económica para ejercerse $\sin$ graves efectos secundarios. En vista de todo ello, el Gobierno del Presidente Suárez promovió los Pactos de la Moncloa (8-27 de octubre de 1977), por los que Gobierno y partidos políticos con representación parlamentaria acordaron un paquete de medidas de ajuste económico, además de otras de carácter político propias de la circunstancia. Para la Seguridad Social, se adoptó el objetivo de reducir costes, así como algunas medidas concretas, una de ellas concerniente a los Servicios Sociales: "Se presentará al Congreso un proyecto de Ley que contemple la reestructuración de las actuales Entidades Gestoras de la Seguridad Social de acuerdo con los principios de simplificación, racionalización, ahorro de costes, eficacia social y descentralización. Asimismo se pasarán a la Administración del Estado el Servicio de Empleo y Acción Formativa, Promoción Profesional Obrera, Instituto Español de Emigración y Servicio de Universidades Laborales, así como el Servicio de Seguridad e Higiene, excepto en las funciones que éste realice de medicina preventiva laboral" (VI.A).

El Real Decreto-Ley citado llevó a términos jurídicos esos acuerdos, adoptando una estructura funcional -salvo en lo que se refiere al Instituto Social de la Marina (disposición adicional tercera)-, que vino a superar la acumulación histórica de órganos de gestión de la Seguridad Social.' Para lo que aquí se trata, la principal medida fue la crea- 
ción, con carácter de entidad gestora de la Seguridad Social, del Instituto Nacional de Servicios Sociales -al que pronto se denominaría abreviadamente INSERSO- "para la gestión de servicios complementarios de las prestaciones del Sistema de Seguridad Social" (art. 1.3). La regulación de su estructura y competencias se llevó a cabo por el Real Decreto 1856/1979, de 30 de julio, en el que dispone que "El Instituto ejercerá su acción fundamentalmente a través de los Servicios Sociales de la Tercera Edad, de Minusválidos Físicos y Psíquicos y de Programas Especiales" (art. 1.2). No aborda la norma las cuestiones sustantivas de tales servicios, pero cabe entender que los dos primeros son los preexistentes de Asistencia a Pensionistas, con nueva denominación, y de Rehabilitación y Recuperación de Minusválidos, con nombre abreviado. En cuanto a éste, la norma citada no dice nada que permita vincularlo al Servicio Social para Inválidos anunciado inicialmente.

Como quiera que la norma creadora del INSERSO no regula el nuevo servicio social que enuncia, no hay ninguna novedad institucional sustantiva que analizar.

\section{LA TRISEGURIDAD SOCIAL DE 1978; SU NIVEL NO CON- TRIBUTIVO}

Cuando se aprueba la Constitución, el gran sistema social protector era de carácter profesional, es decir, destinado a la población trabajadora -bien que en sentido amplio-y a sus beneficiarios. Su régimen de protección era automático, esto es, independiente de los estados de necesidad y con financiación básicamente contributiva, vale decir, procedente en altísima proporción de las cotizaciones de empresarios y trabajadores. La Constitución adoptó una fórmula que entrañaba novedades: "Los poderes públicos mantendrán un régimen público de Seguridad social para todos los ciudadanos, que garantice la asistencia y prestaciones sociales suficientes ante situaciones de necesidad, especialmente en caso de desempleo. La asistencia y prestaciones complementarias serán libres" (art. 41). Son varias las opciones que abre esta fórmula, comenzado por la institución de un primer nivel universal adscriptivo o de renta básica garantizada. ${ }^{9}$ Pero se prefirió construir

7 Ver Manuel Aznar López, "Una institución centenaria: La Seguridad Social española", op. cit., pp. 86 y 87.

8 Ver Manuel Aznar López y Demetrio Casado, Perspectivas de la Seguridad Social española, Acebo, Madrid, 1988, p. 39.

9 Ver Philippe Van Parijs, "Basic income: Guaranted Minimum Income For The $21^{\text {st }}$ 
conservando el núcleo de la Seguridad Social profesional contributiva, al que se han sumado dos ampliaciones. ${ }^{10}$ Para atender las situaciones de necesidad, se provee un nivel asistencial. ${ }^{11}$ Para dar cauce a la protección libre, se regula la vía privada -que dejo fuera de esta exposición-.

La Ley 13/1982, de 7 de abril, de integración social de los minusválidos (LISMI) encomienda al Gobierno la regulación de un "Sistema de prestaciones sociales y económicas" para el tiempo que se demore el desarrollo de la Seguridad Social del artículo 41 de la Constitución (art. 12.1). Este sistema abarca: a) "Asistencia sanitaria y prestación farmacéutica", b) "Subsidio de garantía de ingresos mínimos", c) "Subsidio de ayuda de tercera persona", d) "Subsidio de movilidad y para gastos de transporte", e) "Recuperación profesional" y f) "Rehabilitación médico-funcional" (art. 12.2). Fueron reguladas estas prestaciones por Real Decreto 383/1984, de 1 de febrero. Las dos de carácter sanitario se abrieron a quienes, estando afectados de minusvalía, carecieran de cobertura sanitaria por la Seguridad Social (arts. 5.b y 8.b). Para las otras se exige además el requisito de pobreza (art. 32), de modo que se les dio carácter de Asistencia Social pública. Por cierto que la referencia a las rentas familiares que establece el Real Decreto para la medición del nivel de ingresos fue recurrida por la FEAPS, argumentando que alteraba el carácter personal que la LISMI atribuía a las prestaciones económicas. El Tribunal Supremo dictó sentencia favorable, de fecha 10 de abril de 1986.

La Ley 26/1990, de 20 de diciembre, declara expresamente que amplía el campo de aplicación y que extiende el ámbito protegido de la Seguridad Social (art. 1), lo que supondrá la generalización, por una parte, de la asignación económica del Servicio Social de Asistencia a Subnormales y, por otra, del anticipo de la LISMI en lo que atañe a prestaciones económicas. Para la población no protegida por la Seguridad Social, establece la Ley pensiones no contributivas de invalidez y de jubilación (art. 2). El acceso a las mismas -que, obviamente, no se vincula a requisitos generales de cotización- viene limitado por el de

Century?", en Polices and instruments to fight poverty in the European Union: The guarantee of a minimum income, IDS, Lisboa, 2000.

10 Ver José Luis Tortuero Plaza, "La evolución de la Seguridad Social española en el periodo postconstitucional; la conformación de un sistema mixto en un tiempo de reforma y crisis", en Una parte de la historia de España. La Seguridad Social, Instituto Nacional de Seguridad Social, Madrid, 2001.

1 Sobre la asistencialización de los derechos sociales para poblaciones económicamente precarias en la Unión Europea ver Gregorio Rodríguez Cabrero, "La política social en España", en Miguel Juárez (director), V Informe sobre la situación social de España, Fundación Foessa, Madrid, 1994, p. 1445. 
pobreza relativa, que se define mediante dinteles de renta. Se trata pues de una Seguridad Social para estados de necesidad y sin base contributiva, que se ha dado en llamar asistencial. En el orden orgánico, la Ley 26/1990 asigna al INSERSO, o a las Comunidades Autónomas a las que se hubieran transferido los servicios del mismo, la gestión de dichas pensiones no contributivas (disposición adicional 4.1). Además, la Ley 26/1990 modifica las prestaciones familiares por hijo a cargo (art. 2) y regula "una asignación económica de régimen contributivo y no contributivo" por cada hijo menor de dieciocho años o afectado por una minusvalía en un grado igual o superior al sesenta y cinco por ciento "a cargo del beneficiario". Esta prestación se reserva a las familias de rentas bajas (art. 3-168.1.a y 2.c).

Por supuesto, el contenido sustantivo de las prestaciones no contributivas reseñadas serían incorporadas al Texto Refundido de la Ley General de la Seguridad Social, aprobado por Real Decreto Legislativo de 1/1994, de 20 de mayo. De este modo se efectúa formalmente la suma del régimen asistencial al de aseguramiento en la Seguridad Social constitucional. También recibe esta Ley la ampliación competencial del INSERSO para dichas prestaciones por invalidez y jubilación (art. 57.1c).

Para calibrar el alcance material de la operación, resulta necesario advertir que las pensiones no contributivas, salvo en lo que se refiere a su vinculación a la Seguridad Social, no son nuevas, sino una reconversión de sus equivalentes del Fondo Nacional de Asistencia Social y de la LISMI, con una importante novedad respecto a la de ingresos mínimos establecida por esta última. La Ley 26/1990 neutraliza el efecto de la sentencia del Tribunal Supremo antes citada al establecer que la renta de referencia para acceder a la pensión no contributiva de invalidez será la familiar, no la propia de la persona con minusvalía (art. 2-137-bis). Sin duda, para completar el modelo familístico de protección a las personas con minusvalía, la Ley 26/1990 extiende el derecho de las prestaciones familiares por hijo a cargo a los hijos mayores de dieciocho años afectos de aquella (art. 3-167.1.a). A propósito de las prestaciones por hijo a cargo, procede indicar que la Ley declara incompatible su percepción con la de otras análogas, como eran las asignaciones del Servicio Social de Asistencia a Subnormales, cuya normativa específica fue derogada (disposición derogatoria).

\section{DEL INSERSO AL IMSERSO ${ }^{12}$}

La primera reglamentación del INSERSO se produce por el Real Decreto $1856 / 1979$, de 30 de julio, posterior a la Constitución pero vin- 
culado a un Real Decreto-ley inmediatamente anterior a la misma. La siguiente regulación del Instituto Nacional de Servicios Sociales se llevó a cabo mediante el Real Decreto 1433/1985, de 1 de agosto, el cual trae la novedad de que concreta el objeto del Servicio Social de Programas Especiales previsto en la norma de 1979 (art. 1.2) en "la población marginada" (art. 1.2). Esta exposición no puede dar razón del fundamento de esta opción, pero el caso cierto es que no se siguió de ella ninguna consecuencia práctica. ¿Influiría en esta inconsecuencia el temor a contrariar las por entonces ambiciosas aspiraciones competenciales de las Comunidades Autónomas en materia de servicios sociales? Otro paso equívoco que cabe citar es la creación, por Orden de 15 de marzo de 1989, del Servicio de Termalismo Social "complementario de las prestaciones del sistema de la Seguridad Social"; criatura que aparece degradada a mero programa en el Real Decreto 140/1997, de 31 de enero de 1997 (art. 4.2).

Dado el carácter formal del Texto Refundido de la Ley General de la Seguridad Social de 1994, no cabe esperar de ella más novedades concernientes al INSERSO que las procedentes de las leyes refundidas; en este caso, su ampliación de competencia a la gestión de las prestaciones no contributivas por invalidez y jubilación. Aparte de esto, vamos a ver el modo como recibe el Texto Refundido el fondo histórico relativo a Servicios Sociales y Asistencia Social. La acción protectora incluye "Las prestaciones de servicios sociales que puedan establecerse en materia de reeducación y rehabilitación de inválidos y de asistencia a la tercera edad, así como en aquellas otras materias que se considere conveniente" (art. 38.1e). En otro precepto se abunda en la concepción tradicional de los servicios sociales -con iniciales minúsculas en esta norma- de "complemento de las prestaciones correspondientes a las situaciones específicamente protegidas por la Seguridad Social" (art. 53).$^{13}$ Se formula también en términos tradicionales la asistencia social discrecional (art. 38.2), si bien se prevén algunos supuestos de aplicación específicos (art. 55.1).

Después de la refundición legal del 94, se acordó un cambio del INSERSO aparentemente importante. Mediante el Real Decreto 140/ 1997, de 31 de enero, cuyo objeto primero fue la modificación de la estructura del Ministerio de Trabajo y Asuntos Sociales, se transforma

12 Sobre los asuntos problemáticos de este epígrafe recibí muy estimables opiniones de José María Alonso Seco y Manuel Aznar López; en todo caso, el texto que sigue, como el resto del trabajo, no pretende alcanzar el difícil arte de la interpretación jurídica.

13 Se ha entendido por algunos analistas que esta calificación es contradictoria con la regulación del art. 38: ver Manuel Aznar López, "Aspectos jurídicos de los servicios sociales: de la referencia constitucional a la legislación ordinaria", Revista del Ministerio de Trabajo y Asuntos Sociales. Asuntos Sociales, n 30, pp. 60 y 61. 
el Instituto Nacional de Servicios Sociales en Instituto de Migraciones y Servicios Sociales; de ahí, el nuevo acrónimo IMSERSO. Manteniendo su condición de entidad gestora de la Seguridad Social, al IMSERSO se le asignan dos bloques de competencias. Por una parte: "la gestión de las pensiones de invalidez y jubilación en sus modalidades no contributivas, así como de los servicios complementarios de las prestaciones de la Seguridad Social para personas mayores y personas con discapacidad, el seguimiento del Plan Gerontológico y del Plan de acción para personas con discapacidad, dentro de las competencias del Ministerio de Trabajo y Asuntos Sociales, y la asistencia técnica a los programas de cooperación internacional en el ámbito de mayores y discapacitados." Por otro lado: "la asistencia a las migraciones interiores, la promoción e integración social de migrantes, la asistencia a los solicitantes de asilo y la promoción e integración social de los refugiados y desplazados" (art. 4.2).

Habiéndose aprobado el Texto refundido de la Ley General de la Seguridad Social, cabe preguntarse si no hubiera resultado procedente, antes de dictar un Real Decreto orgánico, reglamentar el contenido y régimen de los servicios sociales de la Seguridad Social. Al no haberlo hecho, se plantean ciertas dudas sobre esa cuestión sustantiva: siendo, como acabamos de ver, que la Ley General incluye, entre las prestaciones de servicios sociales que puedan establecerse, la "materia de reeducación y rehabilitación de inválidos", ¿ son reductibles a la misma los "servicios complementarios de las prestaciones de la Seguridad Social para... personas con discapacidad" asignados a la competencia del IMSERSO en el Real Decreto de 1997? También resulta del caso preguntarse si la habilitación del IMSERSO para ocuparse de la inmigración implica la conversión del contenido de esa nueva competencia en un "complemento de las prestaciones correspondientes a las situaciones específicamente protegidas por la Seguridad Social", según caracteriza los servicios sociales de la misma la Ley General de 1994 (art. 53). La razón de esta pregunta consiste en que, si bien la Ley General de la Seguridad Social abre la posibilidad de nuevos servicios sociales (art. 38.1b), no parece claro que ciertas atenciones a inmigrantes, refugiados, desplazados y demandantes de asilo tengan el carácter complementario que exige la propia Ley.

Sin posteriores novedades reglamentarias relativas a las cuestiones sustantivas de los servicios sociales de la Seguridad Social, se ha producido una nueva norma orgánica: el Real Decreto 238/2002, de 1 de marzo. Esta norma mantiene el núcleo de competencias delimitado por su precedente, salvo que acusa algunas mermas en materia de migración, como resultado del reparto de cometidos con la Delegación del Gobierno para la Extranjería y la Inmigración. En sentido contrario, 
aparecen algunas competencias nuevas, pero de carácter aparentemente instrumental. El objeto propio, no condicionado por circunstancia externas, del Real Decreto, como puede leerse en su exposición de motivos, es la sustitución de la organización interna por colectivos por otra referida a funciones.

$* * *$

Entre los servicios sociales de la Seguridad Social efectivamente desarrollados, los destinados a -según se usa decir ahora- personas mayores y con discapacidad tienen carácter selectivo en función, no de insuficiencia de rentas personales, sino de las necesidades especiales asociadas a las citadas circunstancias personales. El contenido de dichos servicios sociales, en términos generales, se aproxima mucho al propio de la rama de intervención social de su propia denominación. Comenzaron teniendo una financiación básicamente contributiva para derivar hacia la fiscal. En cuanto a su técnica institucional de protección, las normas reglamentarias parecen privilegiar a los órganos de gestión en perjuicio de las figuras prestacionales enunciadas por las leyes, de modo que las mismas vienen a derivar en poco más que habilitaciones administrativas de carácter orgánico. 